\title{
A COMPUTATIONAL MODEL OF THE ROLE OF IONIC-LOCK INTERACTIONS IN LIGAND RECOGNITION BY THE HUMAN C3a RECEPTOR
}

\author{
VOLETI S.R. \\ Institute of Life Sciences, University of Hyderabad Campus, Gachibowli, Hyderabad- 500046, AP, India. \\ *Corresponding Author: Email- sreedhara.voleti@gmail.com
}

Received: May 22, 2012; Accepted: May 31, 2012

\begin{abstract}
Computational techniques such as homology modeling and molecular dynamics were used to identify novel intramolecular ioniclock interactions among key charged residues spanning the transmembrane helices and extracellular loops of human complement C3a receptor and the synthetically prepared human C3a receptor ligand SB290157. The results shed light on the active site of the human C3aR and the arginine specificity of the ligand. A strong ionic-lock region with interactions among the charged residues R161 (R4.64), E162 (ER4.65), R340 (R5.42), K96 (K3.36), D167 (D4.70), D417 (D7.35) and H418 (H7.36) is critical for the structural integrity of human C3aR. Results of docking both the stereoisomer of SB290157 with human C3aR indicate that the active site consist of the aromatic residues F107 (F3.37), Y160 (Y4.63), F345 (F5.47), W390 (W6.48), Y393 (Y6.51) and F396 (F6.54) together with the ionic-lock region and reveal the lack of stereospecific preference of human C3aR for SB290157. The active site features of human C3aR and the proposed ligand-binding region may permit the discovery of novel antagonists for $\mathrm{C} 3 \mathrm{aR}$ as potential therapeutic agents for conditions such as asthma.
\end{abstract}

Key words- homology modeling, molecular dynamics, lonic-lock interactions, Ligand, therapeutic agents.

Short Title- lonic-lock interactions of charged residues in C3aR and the ligand recognition

Citation: Voleti S.R. (2012) A Computational Model of the Role of lonic-Lock Interactions in Ligand Recognition by the Human C3a Receptor. International Journal of Drug Discovery, ISSN: 0975-4423 \& E-ISSN: 0975-914X, Volume 4, Issue 1, pp.-128-136.

Copyright: Copyright@2012 Voleti S.R. This is an open-access article distributed under the terms of the Creative Commons Attribution License, which permits unrestricted use, distribution and reproduction in any medium, provided the original author and source are credited.

\section{Introduction}

G protein-coupled receptors (GPCRs) are membrane bound proteins involved in he first phase of cellular signal transduction leading to diverse functional effects. GPCRs have been classified into 3 distinct families (scheme-1). The structure of the Type-1 family of GPCRs became clearer from the crystal structures of bovine and bacterial rhodopsin and consists of 7 transmembrane helices joined by three extracellular and three intracellular loops. The extracellular $\mathrm{N}$-terminal residues are connected to the first transmembrane (TM1) helix, while the intracellular C-terminal residues attach to the seventh transmembrane (TM7) helix. Recognition of endogenous ligand by GPCRs is mediated by the extracellular Nterminal chain of residues, while the primary signal transduction mechanism occurs through induction of a conformational change in the transmembrane helices, intracellular loops and the Cterminus. During the conformational change, disruption of saltbridge interactions alters the function of the receptor, as already noted in the interaction of a lysine and aspartic acid in transmembranes 3 and 7 and $\alpha-1$ adrenergic receptors [1-3]. Modulation of such interactions has led to the discovery of novel ligands for use in drug discovery. Plethora of research data is available in the homology modeling of G-protein coupled receptors (www.gpcr.org). Fowler [4] presented an overall strategy for drug discovery in the context of modeling GPCRs.

Human C3a receptor (C3aR) is a Type-1 GPCR that contains a single chain of 482 aminoacids (Fig. 1). Human C3aR has an unusually large second extracellular loop (EC45) ( 180 amino acids); its function was established through mutation studies [5] which revealed that deletion of a large number of residues (aminoacids 198-308) did not lead to any appreciable loss in function. Deletion of residues (aminoacids 182-197 and 309-331) from the second extracellular loop (EC45) led to a significant decrease in the binding affinity of the natural ligand C3a to the human C3aR 
[5]. These results indicate that only a few residues at the helixloop junction of the receptor appear to be involved in key C3aR$\mathrm{C} 3 \mathrm{a}$ interactions. Mutation of the acidic residues at the junctions of TM4 and TM5 with EC45 significantly decreased the binding of C3a. It may be assumed that a multiple point mutation of aspartic acid residues (D183K/D186K/D325K/D326K/D327K) located at the junction of TM4 and TM5 with EC45 results in a loss of binding of $\mathrm{C} 3 \mathrm{a}$ by a change in the receptor conformation and loss of ligand -binding contacts [5].

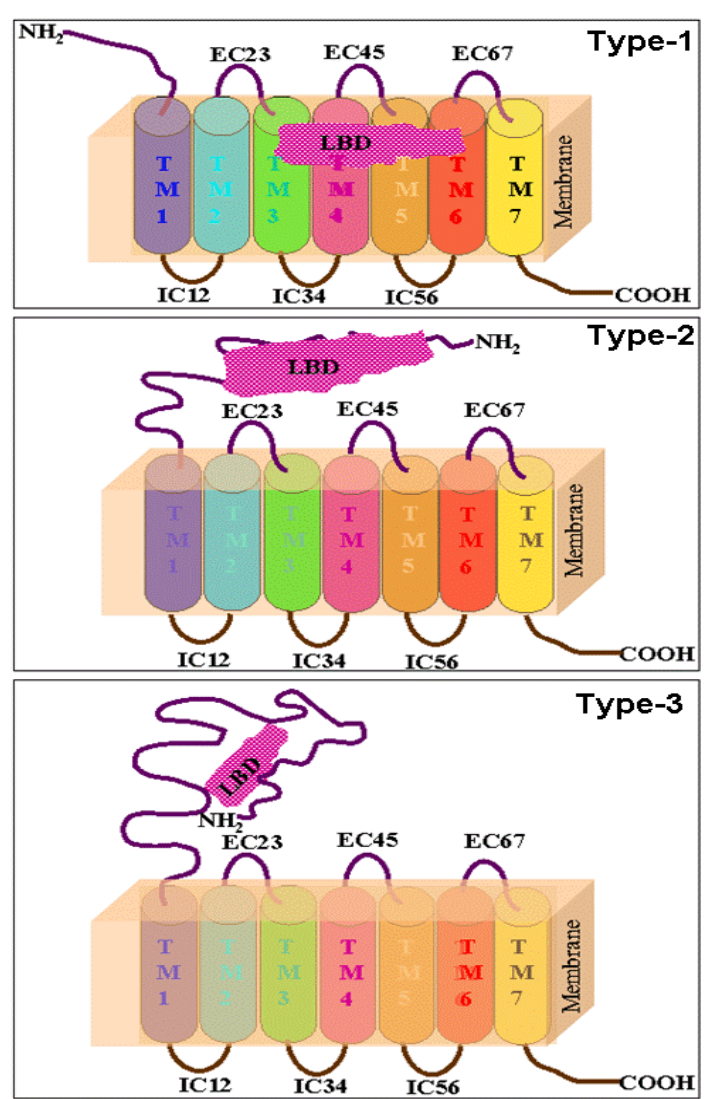

Scheme 1- Schematic representation of the three different families of GPCRs. Ligands bind to different regions (LBD) of the different families of GPCRs. Transmembranes are shown as cylinders connected by intracellular and extracellular loops.

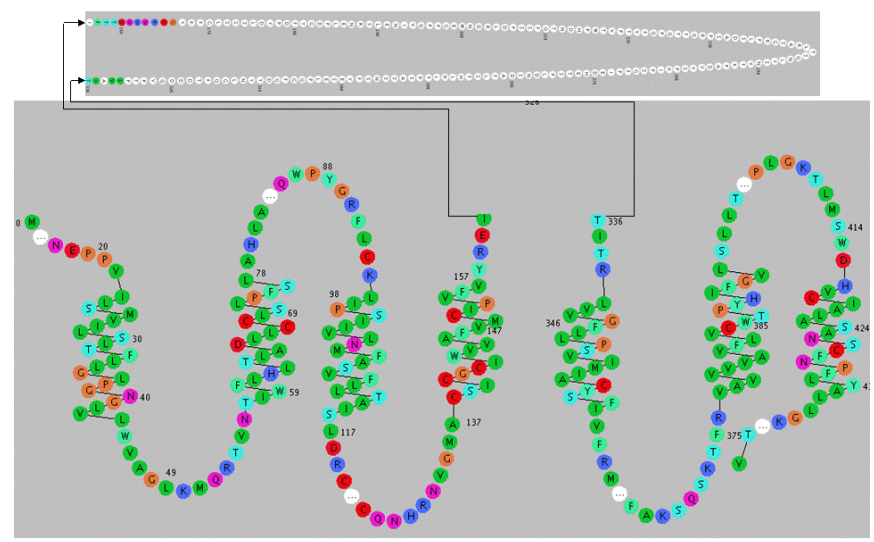

Fig. 1- Snake plot of human $\mathrm{C} 3 a \mathrm{R}$. Residues in color indicate features such as acidic, basic, polar and hydrophobic.
The compliment cascade controls the immune and inflammation response. When the compliment cascade is activated upon exposure to allergens, the complement mediator $\mathrm{C} 3 \mathrm{a}$ is released which binds to the natural C3aR receptor expressed in mast cells, eosinophils and basophils. The resulting release of inflammatory mediators manifests as smooth muscle contraction, which in lungs results in an asthmatic condition [6-12]. Role of C3a in inflammation diseases such as asthma is yet to be rationalized, but the role of $\mathrm{C5a}$, another anaphylatoxin is well characterized in diseases like sepsis and other immune-related inflammation lung injury. Recent work by Thangam, et al [13] showed significant increase in the expression of $\mathrm{C} 3 a$-induced mast cell degranulation upon incubation of human mast cells with ASM cells. This finding led to the postulation of a role of $\mathrm{C} 3 \mathrm{a}$ and $\mathrm{C} 3 \mathrm{aR}$ in asthma. However, since the results were based on experiments using the human mast cell line LAD2, which was derived from a patient with mast cell leukemia, the relevance of the results to asthma may be found questionable [14].

C3a is a single chain 77-aminoacid peptide with a C-terminal arginine. There are six cysteinyl residues in C3a forming 3 disulfide bonds and whilst the terminal regions of the peptide form $\alpha$ helices [15-16]. C3a induces histamine-mediated contraction of guinea pig ileal tissue in vitro and at much lower doses increases the vascular permeability of human skin in vivo [17]. A conceptual model has been presented on how the $\mathrm{C}$-terminal arginine residue interacts with the human C3aR [17]. Even though there was no direct evidence for critical residues of human C3aR being involved in the binding of $\mathrm{C} 3 \mathrm{a}$, it is logical to assume that a salt-bridge interaction exists between $\mathrm{C} 3 \mathrm{a}$ and $\mathrm{C} 3 \mathrm{aR}$. It has been shown earlier that $\mathrm{C} 3 \mathrm{a}$ is rapidly inactivated in plasma by the action of carboxypeptidase through removal of the carboxy terminal arginine residue [18]. The resulting 76-residue peptide exhibits no inflammatory activity and indicates that Arg77 is critical and essential for activity. Similarly, series of short pentapeptides with arginine as a Cterminal residue have been shown to be significantly active [19]. Site directed mutagenesis studies by Sun, et al [20] indicated those residues likely to be involved in $\mathrm{C} 3 a$ binding to human C3aR. Particularly, mutation of R161 (R4.64), R340 (R5.42) and D417 (D7.35) altered significantly the binding affinity of C3a, while mutation of $\mathrm{K} 96$ (K3.36) and $\mathrm{H} 81$ had only a minor effect. A 3dimensional model of interactions of human C3aR (developed from the neuropeptide $Y 1$ receptor template) with $\mathrm{C} 3 \mathrm{a}$ has been proposed [20], but detailed computational analysis of the position of the key mutated residues has not been discussed at length. Further detailed computational analysis on the position of the key mutated residues was not discussed at length. In a constant endeavor to develop drugs as potential therapy for asthma, several pharmaceutical companies have been involved in developing antagonists of C3aR and Ames, et al [21] prepared SB290157 (shown in Scheme-2) as a competitive inhibitor of human C3aR expressed in RBL (IC50 200nM). Such antagonists are nonpeptidic but contain an arginine moiety to be recognized by the C3aR. SB290157 inhibited both C3a-induced Ca2+ release and human neutrophils in functional assays. A recent article by Mathieu, et al [22] has suggested that SB290157 possess more agonistic activity in some functional assays thus giving more intriguing data. 


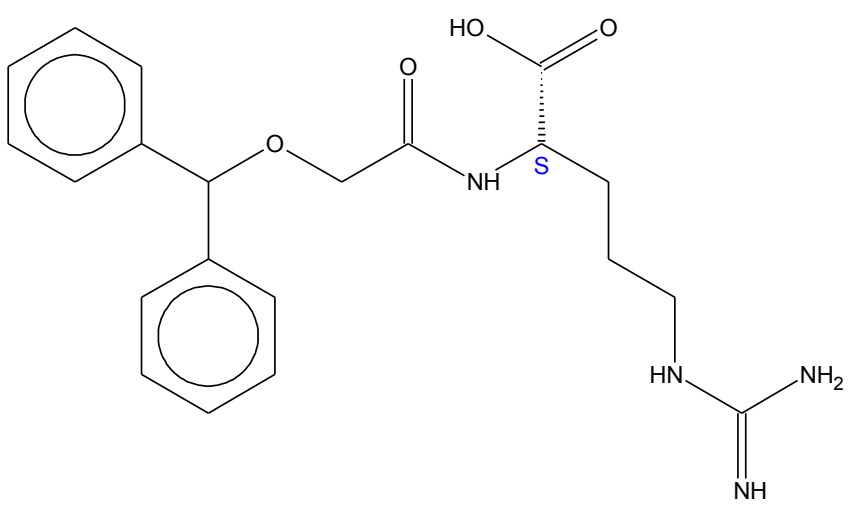

Scheme 2- Schematic representation of SB290157

We have used homology modeling and molecular docking techniques to develop a 3-dimensional structural model of human C3aR; to elucidate its putative active site; and to identify how ligands bind within the active site. Our focus was to identify the orientation of the key residues in $\mathrm{C} 3 \mathrm{aR}$ which altered the nature of binding of $\mathrm{C} 3 \mathrm{a}$ as revealed by the mutational and experimental data $[5,20]$. The resulting hypothesis of intramolecular salt-bridge interactions was extended to the binding of both stereoisomers of SB290157.

\section{Computational Methods \\ Homology model of human C3aR}

The 3-dimensional coordinates of Bovine Rhodopsin (1HZX) [23] were taken as an initial template on which to build the homology model of human C3aR [NP_004045 NCBI, Q16581 Swiss-Prot]. The transmembrane residues of $\mathrm{C} 3 \mathrm{aR}$ were aligned on to the coordinates of the transmembrane domain of bovine rhodopsin to give a coarse 3-dimensional TM structure of C3aR using maximal homology. Fig. 2 gives the sequence alignment of the transmembranes of bovine rhodopsin and C3aR. The nomenclature used for the residues of the transmembrane is that of Weinstein, et al. $[24,25]$. Initially, highly conserved residues of the bovine rhodopsin [N1.50 (N41), D2.50 (D68), R3.50 (R120), W4.50 (W147), P5.50 (P348), P6.50 (P392) and P7.50 (P432)] were matched to the corresponding residues in each transmembrane of $\mathrm{C} 3 \mathrm{aR}$.

\begin{tabular}{|c|c|}
\hline 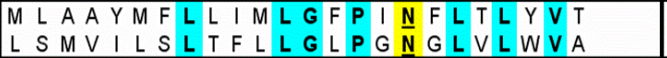 & human_C3aR_TM1 \\
\hline 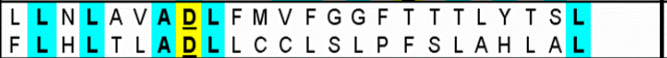 & $\mathbf{m L}$ \\
\hline 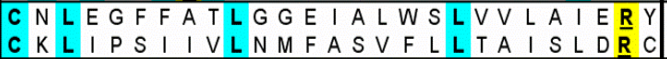 & \\
\hline 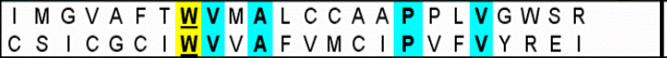 & TM4 \\
\hline 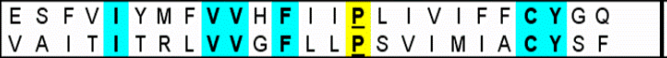 & \\
\hline 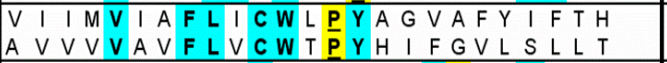 & \\
\hline $\begin{aligned} \text { F M T I P } \\
W D H V C\end{aligned}$ & \\
\hline
\end{tabular}

Fig. 2- Sequence alignment of transmembranes of bovine rhodopsin $(1 \mathrm{HZX})$ and the human C3aR (Residues shown bold, underlined and shaded yellow are the most conserved, while residues in bold and shaded in blue are the most similar).

An initial 3-dimensional homology model structure of the helical domain arrangement of the C3aR was then produced by mutation of the residues of $1 \mathrm{HZX}$ with the corresponding residues of the human C3aR. Information on the N-terminal, extracellular (EC), intracellular (IC) and C-terminal sequence lengths were taken from the website http://www.expasy.org/uniprot/Q16581. Loops were joined to the $\mathrm{N}$-terminal and $\mathrm{C}$-terminal sides of the helices to form a complete single chain using the loop-search algorithm in the Biopolymer module of SYBYLTM [26] and the best possible initial conformation. However, a rational initial loop structure for the second extracellular loop (EC45) could not be obtained owing to its large size ( 180 aminoacids) and so a large loop was created and its $\mathrm{N}$ - and $\mathrm{C}$-terminals attached to TM4 and TM5 with no obvious structural conflicts. Finally, the extracellular N-terminal and intracellular C-terminal sequences were attached to the TM1 and TM7, respectively, to form a single chain of 482 amino acids. A disulfide bridge between the cystine residues 95 (in EC23) and 172 (EC45) was built and hydrogen atoms added to complete the $\mathrm{C}, \mathrm{N}, \mathrm{O}$ and $\mathrm{S}$ valencies. The main problem in building the model lay in finding the orientation of the mutated aspartic acid residues [5], because it was not clear whether they were involved directly in ligand binding, even though they were located at the junction of EC45 with TM4 and TM5. This question was highly critical in obtaining a good initial conformation of the protein.

The coarse full-length 3-dimensional structure of human C3aR with the backbone of the TMs exactly mimicking that of bovine rhodopsin was subjected to a quick energy minimization with the default parameters in SYBYL, together with Tripos Force Field and Gasteiger-Huckel charges. The ProTable module in the biopolymer section of SYBYL was used to eliminate all bad chirality issues in the initial structure and once an acceptable initial orientation of the important residues was obtained, an energy minimization process using Triops force field were carried out to eliminate all bad contacts within the protein. The root-mean-square difference (RMSD) between the initial and final optimized protein was significantly less than $1 \AA$ suggesting minor differences existed between them. The Tripos force field with Gasteiger-Huckel charges was used, therefore, for the remaining computational studies in the gas phase.

Molecular dynamics of the overall initially energy minimized structure was carried out for $100000 \mathrm{fs}$ with a step size of $1 \mathrm{fs}$ with snapshots taken at $100 \mathrm{fs}$ step size. Longer molecular dynamics calculations were necessary owing to the presence of the large 180 amino acid length extracellular loop (EC45) which needed time to settle into a stabilized fold. An average structure of the protein was computed by averaging the coordinates of each atom of the protein over the entire energy equilibrated plateau as a function of time, which was further subjected to energy minimization to produce an initially acceptable 3-dimensional model of human C3aR. All parameters used were default values except for the number of cycles during the energy and structure optimization process, which was set to 100000 . All molecular dynamics and energy minimization studies were performed in the gas-phase only.

\section{Docking of SB290157 to identify the active site}

An approximate active site of human C3aR was located with the help of the MOLCAD module of SYBYL, based on the arginine specificity of the C3a and the available mutation data $[5,20]$. The interaction of C3a was modeled with SB290157, which is an arginine containing analog and the human C3aR. Arginine is a posi- 
tively charged residue which can be stabilized by the complimentary charges offered by the aspartic acid residues of the receptor. Once an approximate position of the arginine moiety of SB290157 was identified within C3aR, the biaryl moiety of the SB290157 was found to lie within the hydrophobic volume of the active site formed by the aromatic residues F107 (F3.37), Y160 (Y4.63), F345 (F5.47), W390 (W6.48), Y393 (Y6.51) and F396 (F6.54).

On completion of manual docking, the protein-ligand complex was energy minimized with the Tripos force field and Gasteiger-Huckel charges. Except for the number of cycles, the default set of minimization parameters were used. Subsequently, molecular dynamics studies were performed on the energy minimized proteinligand complex. This time, molecular dynamics studies were carried out for 50000 fs with 1 fs step size and a 100fs snapshot. An averaged structure of the protein-ligand (C3aR-SB290157) complex was then computed over the energy equilibriated region and further subjected to energy minimization to produce a final proteinligand complex structure. To understand the differences between the stereospecificity of SB290157, molecular dynamic runs were carried out for both the R and S isomers of SB290157. Binding energies were computed from the energies of the free ligand, free protein and the protein-ligand complex, using the Tripos Force Field and Gasteiger-Huckel charges.

\section{Results and Discussion}

A full length 3-dimensional homology model of human C3aR developed from the bovine rhodopsin crystal structure is presented in Fig. 3A. Our focus was to understand the environment and orientation of the residues present in the extracellular loop that joins TM4 and TM5 that were previously mutated $[5,20]$. In addition, we were also interested in the acidic, basic and polar residues which lie at the junction of the extracellular loop (EC45) with the transmembrane helices TM3-TM7. The disulfide bond between the residues C95 (EC23) and C172 (EC45) was found to bring a tighter packing EC23 and EC45 loops and the transmembranes TM3, TM4 and TM5 - thereby stabilizing the large extracellular EC45 loop and enhancing the receptor folding (Fig. 3A inset). Both the cystine residues (C95 and C172) are located near the junction of extracellular environment and the helices TM3and TM4 (Fig. 1). When the disulfide bridge between $\mathrm{C} 95$ and $\mathrm{C} 172$ was not constructed, TM3 and TM4 moved away during the molecular dynamics as the EC23 and EC45 were no more connected. The close proximity of EC23 and EC45 resulting from the disulfide bridge at the junctions of TM3 and TM4 provides tight packing of the TM domains. It was noticed that without the disulfide bridge, the helices TM3 and TM4 were separated by approximately 2-3 angstroms (RMSD), the consequences of which were not further explored in our homology modeling. Fig. 3B and Fig. 3C represents the view of transmembranes along the membrane and through the extracellular environment, respectively (the extracellular and intracellular loops and $\mathrm{N}$-terminal and $\mathrm{C}$-terminal sequences were not shown for clarity). Fig. $3 \mathrm{C}$ represents a circular orientation of TM2-TM7 with respect to TM3 (Magenta), which is very much similar to that seen for bovine rhodopsin. The RMS deviation of the backbone of transmembranes TM1-TM7 of bovine rhodopsin and human C3aR is approximately $2.3 \AA$ when the most conserved residues are aligned.

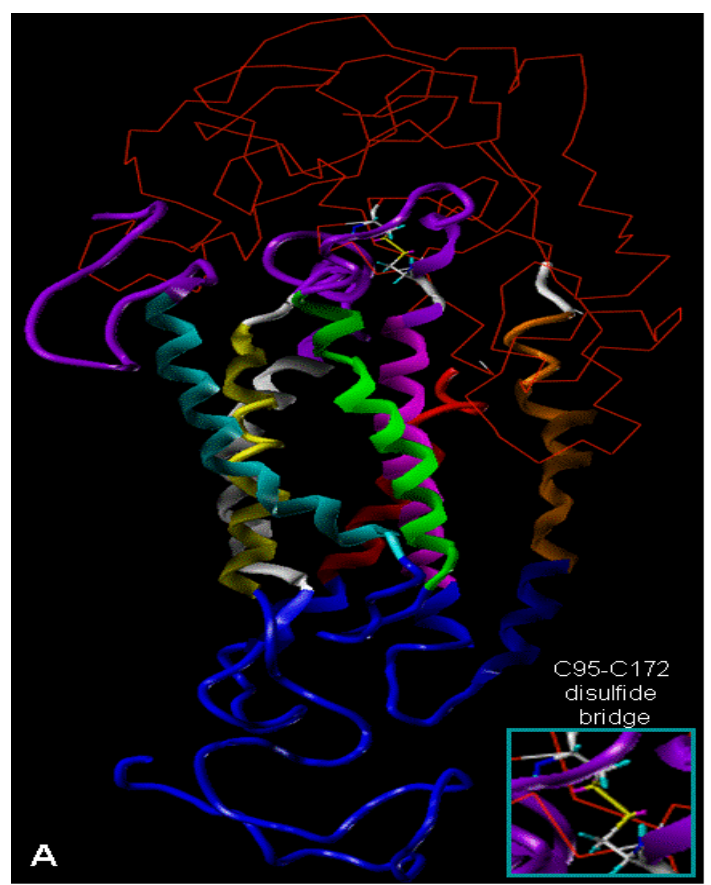

Fig. 3A- Full-length homology model of human C3aR developed from bovine rhodopsin. Color codes in ribbon-tube structural format are TM1-Cyan; TM2-Green; TM3-Magenta; TM4-Orange; TM5-Red; TM6-White; TM7-Yellow; N-terminal and extracellular environment - Violet; C-terminal and intracellular environment Blue. Large extracellular EC45 loop is shown as thin line in Red. Disulfide-bridge between C95-C172 (represented as a Yellow stick) is shown as an inset.

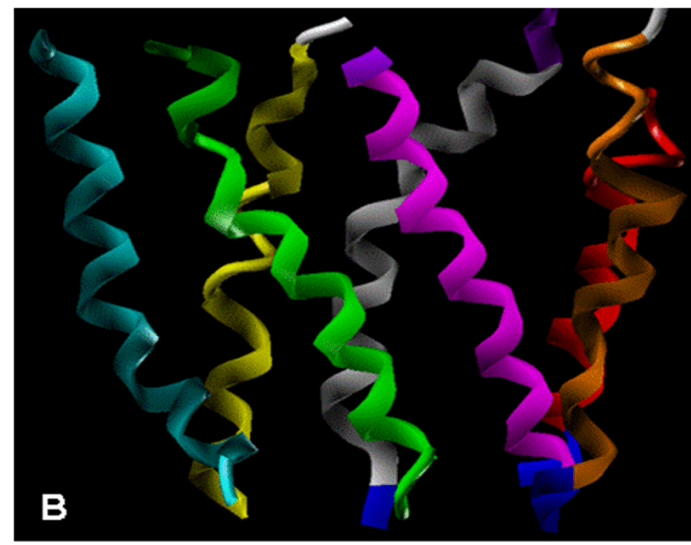

Fig. 3B- View of the transmembranes of human C3aR along the membrane with the extracellular and intracellular environment to the top and bottom, respectively.

Closer examination of the aspartic acid residues in the large extracellular loop EC45 which were for which mutations studies were carried out [5] [D183, D186, D325, D326 and D327] revealed several interesting features. It appears that D183 and D186 in EC45 are located far from the C-terminal junction of TM4 and are stabilized by hydrogen bonding interactions with the backbone. Moreover, there are no surrounding positively charged residues which could stabilize the aspartic acid residues (D183 and D186) in a salt-bridge fashion. The interatomic distances between the charged carboxylate oxygen atoms of the aspartic acid triad 
(D325, D326 and D327) and the positively charged residues R248 and K411 (K7.29) suggest significant ionic-lock interactions (distances $<2 \AA$ ) as shown in Fig. 3D. R248 is located close to the mutated aspartic acid residues, while K411 (K7.29) resides very close to the interface of the N-terminal of TM7 and the extracellular loop (EC45). Although D325 has a strong salt bridge interaction with R248, it is also possible that D326 has a similar interaction with R248 because EC45 is a large loop of approximately 180 amino acids with no structural integrity from molecular modeling studies. K411 (K7.29) stabilizes D327 of EC45 through ionic-lock interactions, which in turn is stabilized by $\mathrm{H}$-bonding with the backbone of E406 and V329. Strong intramolecular ionic-lock interactions between the aspartic acid triad and R248 and K411 (K7.29) suggests that the mutation of these triad aspartic acid residues may lead to a conformational change of EC45, thereby causing a significant reduction of $\mathrm{C} 3 \mathrm{a}$ binding to human C3aR. The conserved residue R120 (R3.50) was found to be stabilized by the cation-hydrophobic interactions, while D119 (D3.49) made strong hydrogen bonding interactions with the residues N57 (N2.39) and N135 (N4.38) at the bottom of the TM3 (as shown in Fig. 3E), with interatomic distances much shorter than conventional hydrogen-bonds. It is suggested that the contribution of several such interactions makes the tertiary structure of the human C3aR more stabilized internally.

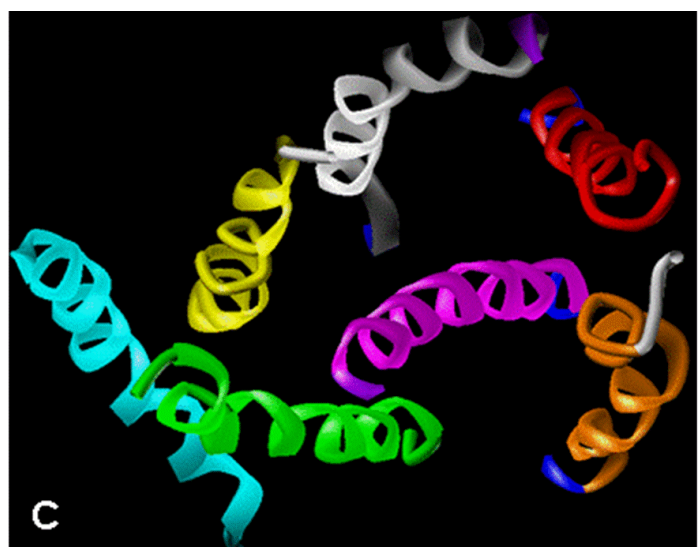

Fig. 3C- Top view (intracellular loops are behind) of the arrangement of the transmembranes of human C3aR.

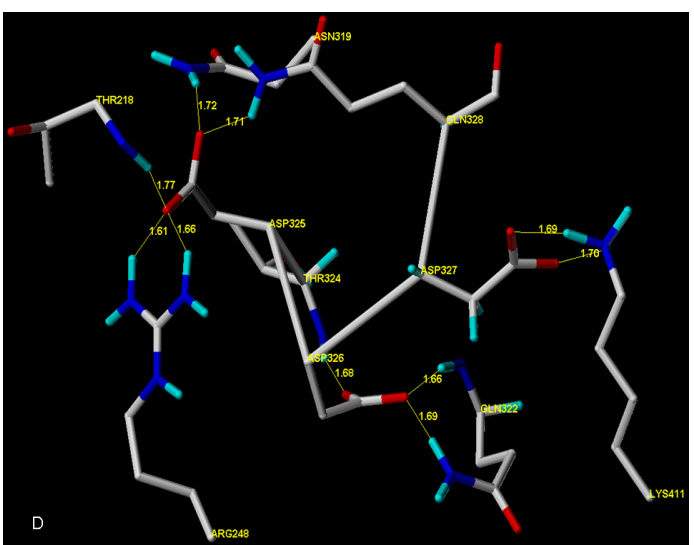

Fig. 3D- lonic-lock interactions between various residues and D325, D326 and D327. Strong hydrogen-bonding and salt-bridge distances among the acidic and basic residues are shown in Yellow. Residue labels are shown in single-letter aminoacid codes.

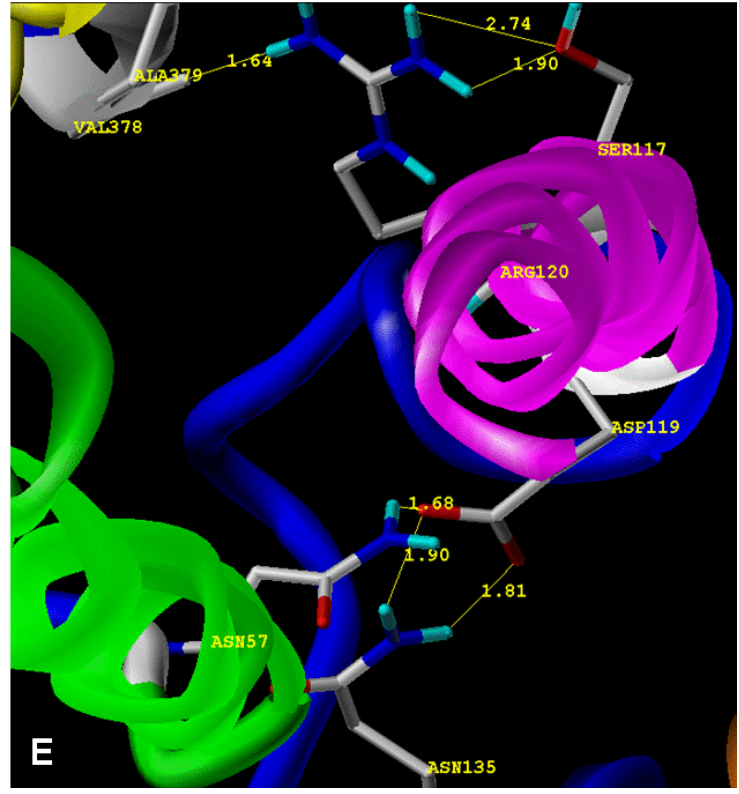

Fig. 3E- Stabilizing interactions of D119 (D3.49) and R120 (R3.50) with surrounding residues in the intracellular environment - Blue. Only TM2 (Green) and TM3 (Magenta) are shown for clarity.

Intramolecular hydrogen-bonding and salt-bridge interactions have been proposed previously for several other GPCRs (such as muscarinic and $\alpha$ - and $\beta$-adrenoceptors) [27-28]. Concrete evidence for such interactions is not available in human C3aR, even though the data published by Sun, et al [see Fig. 5A and B of [20] suggests the orientation of the residues R161 (R4.64), R340 (R5.42) and D417 (D7.35) in the active site represents the presence of complimentary features from surrounding residues. Intramolecular salt-bridge interactions between transmembrane residues and extracellular and/or intracellular loop residues lead to higher stability and structural integrity within the transmembrane domains, whilst salt-bridge interactions within the residues of the transmembrane domains (such as the Asp/Glu residues of TM3 and the corresponding arginine/lysine residues of TM2-TM7) provide additional orientation of these charged residues within the active site. It is likely that such directed orientation of aminoacid residues by strong stabilizing interactions is a pre-requisite for ligand recognition by $\mathrm{C} 3 \mathrm{aR}$. Indeed, it has been shown earlier that disruption of the arginine cage can induce a ligand dependant switch within muscarinic [27] and $5-\mathrm{HT}_{2 \mathrm{~A}}[28]$ receptors.

In our quest to identify key interactions among the charged residues, we noticed upon a prominent intramolecular hydrogenbonding network within the $\mathrm{C} 3 \mathrm{aR}$ involving residues located in the transmembrane region and the adjoining extracellular loop EC45, as illustrated in Fig. 4A. Closer examination of the residues K96 (K3.36), R161 (R4.64), R171, S241, R244, R340 (R5.42) and $\mathrm{H} 418(\mathrm{H7} .36)$ indicated that the residues were not only located at the junction of the extracellular loops with the transmembrane helices but also involved in ionic-lock interactions. These residues appear to be stabilized by the intramolecular salt-bridge pairs [D167-\{K96 (K3.36), R161 (R4.64), R244)\}], [Y174-\{K96 (K3.36)\}], [\{E162 (E4.65)\}-\{Y160 (Y4.63), R340 (R5.42)\}], [E20-R171] and $[\{\mathrm{D} 417$ (D7.35)\}-\{H418 (H7.36), Y393 (Y6.51)\}]. The interactions were maintained throughout the molecular dynamics studies and 
seem, therefore, not to be false positives. Fig. $4 \mathrm{~A}$ and Fig. 4B shows the location of the polar residues involved in the ionic-lock interactions and Fig. $4 \mathrm{C}$ shows the strong ionic-lock interaction distances between the charged residues.
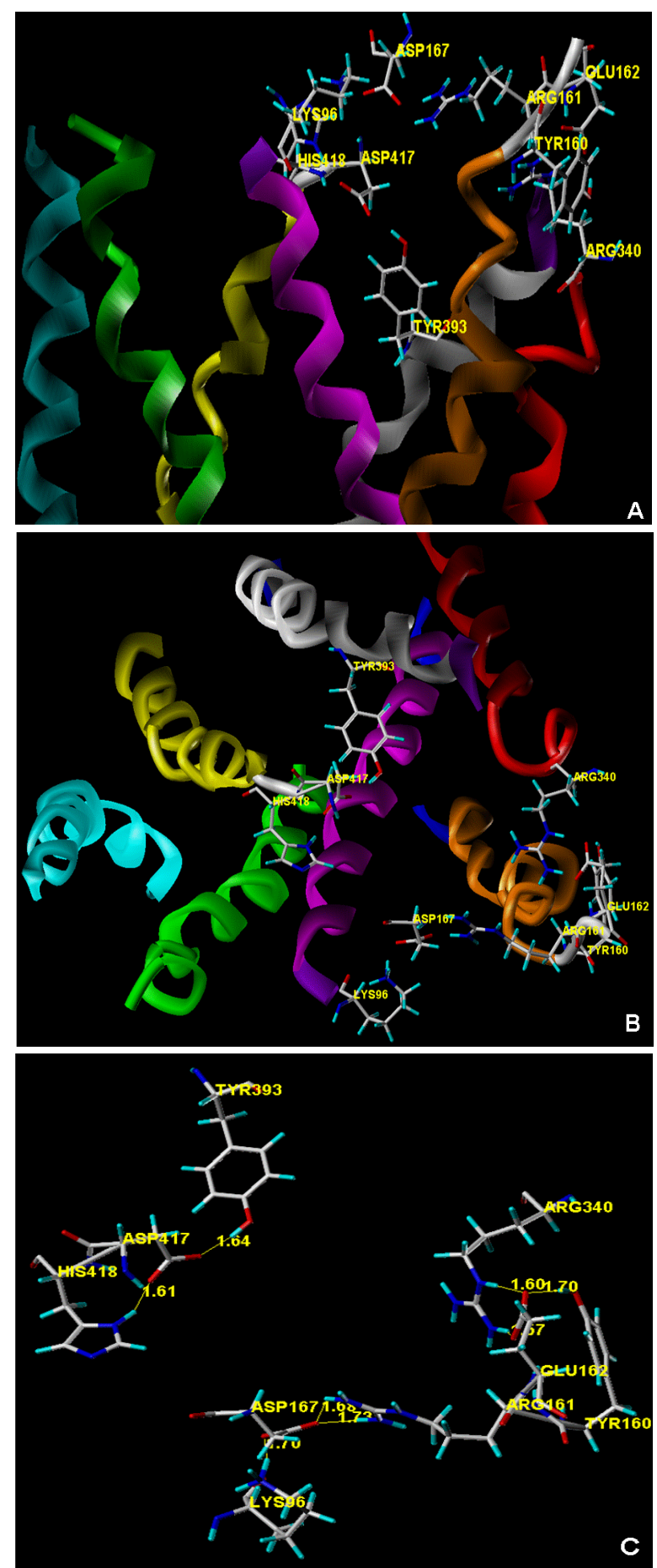

Fig. 4- lonic-lock interactions among key acidic, basic and polar residues at the junction of extracellular loops and transmembrane helices of human C3aR. (A) Side view along the membrane and (B) Top View with intracellular environment lying behind. (C) View highlighting the strong intramolecular ionic-lock distances (in $\AA$ ) among the acidic and basic residues. Helices are not shown for clarity.
It was also observed that a hydrophobic region exists among the residues located 3-4 turns within the transmembranes TM3, TM5, TM6, just below the proposed ionic-lock interactions, as illustrated in Fig. 5. Within TM6, residues F386 (F6.44), W390 (W6.48) and Y393 (Y6.51) were found to form $p$-stacking interactions with each other, which together with the residues F107 (F3.37) and F345 (F5.47) in TM3 and TM5 respectively, form a tight hydrophobic volume. In addition, the residues in TM5 were all pointed in the same direction within the transmembranes, thus creating a hydrophobic volume. This implies the presence of an active site, as illustrated in Fig. 6A, consisting of only one pocket was located. This site was visualized in the area of the hydrophobic and saltbridge interactions by use of the MOLCAD module and Fast Connolly Channel Surface calculations in SYBYL [26]. The shape of the active site located among the transmembranes TM3-TM6 was found to consist of a hydrophobic volume extending deep into the transmembrane region with the electrostatic part located closer to the extracellular surface, as shown in Fig. 6B. Gasteiger-Huckel charges were used to calculate the individual active site features. Although the volume of the proposed active site might vary in reality, the location and the shape of the computed active site was taken as an initial starting point to carry out further molecular modeling studies on the protein-ligand complexes.

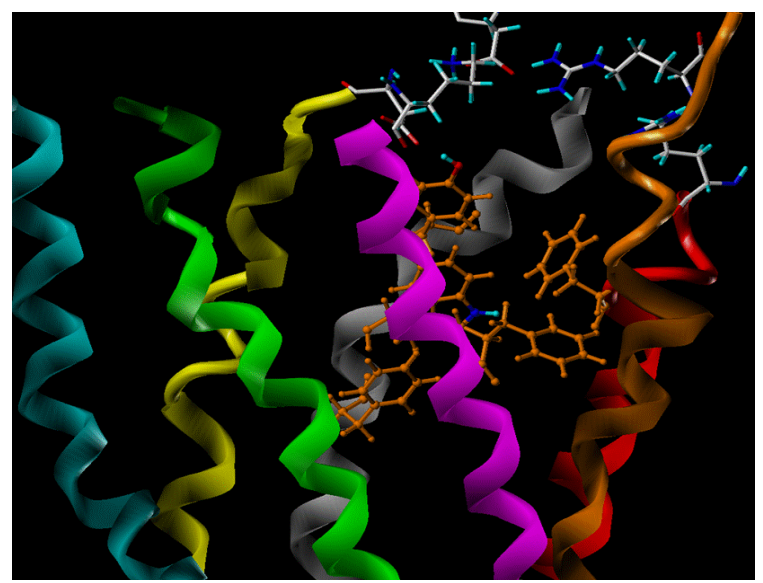

Fig. 5- Hydrophobic volume of the active site of human C3aR comprising the aromatic residues (brown) of the transmembranes TM3 - TM7 lying below the ionic-lock region.

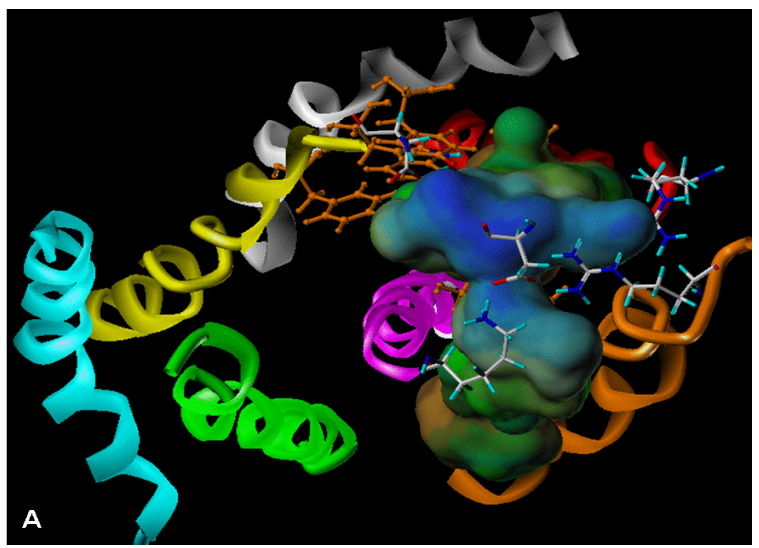

Fig. 6A- Top view of the active site volume and shape of C3aR. Hydrophobic (Brown) and electrostatic (Blue) features of the active site are shown with a top view. 


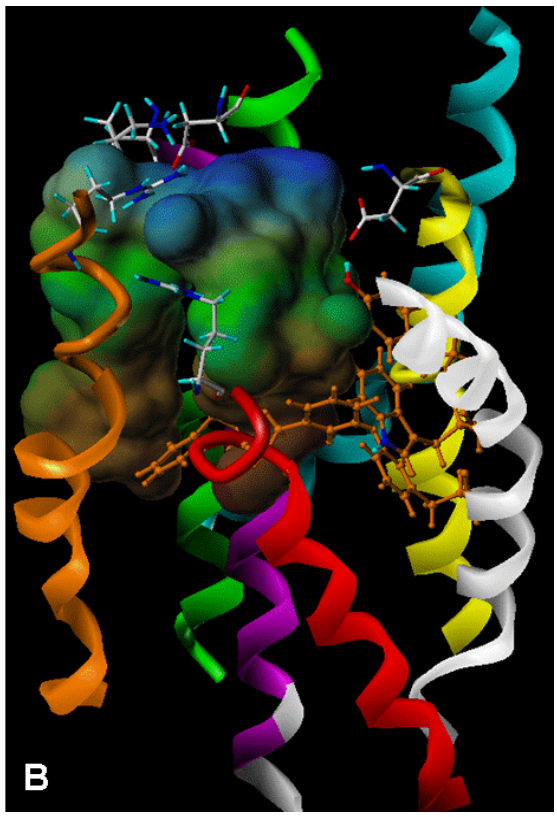

Fig. 6B- Side view of the active site features and the active site volume.

In order to identify the binding mode of SB290157, a conformational search analysis using the random search technique in SYBYL with Gasteiger-Huckel charges was performed. The lowest energy conformation had a bent U-shaped structure, which corroborated with the overall shape of the active site of $\mathrm{C} 3 \mathrm{aR}$. Manual docking of SB290157 into the proposed active site is illustrated in Fig. 7A. The hydrophobic region of the active site was found to bind by the biaryl moiety of SB290157, whilst the carboxylic acid moiety of SB290157 was stabilized by R161 (R4.64) and R340 (R5.42), which in turn were stabilized by D167 (D4.70) and E162 (E4.65), respectively. This implies that the positive charge of the arginine moiety of SB290157 is bound to the D417 (D7.35) via an intramolecular salt-bridge and that the hydrophobic region of the receptor is also stabilized with the strong positive charge generated in SB290157 through cation-p interactions. Moreover, D417 (D7.35) is also stabilized by strong hydrogen bonding with Y393 (Y6.51) and H418 (H7.36) within the human C3aR. Surprisingly, therefore, a whole new sequence of intramolecular saltbridge interactions was found to occur between SB290157 and C3aR. Molecular dynamics for 50,000fs was performed for the ligand-protein complex using Gasteiger-Huckel charges and a $1 \mathrm{fs}$ step size and a 100 fs snapshot interval time. The whole protein and the ligand were permitted to move freely with no constraints applied throughout the molecular dynamics study. It was found that at all stages of the molecular dynamics and energy minimization, that all salt-bridge interactions were maintained strongly and that SB290157 maintained its interactions with human C3aR. The final energy minimized structure computed after averaging the energy-equilibriated region is represented in Fig. 7A.

The critical interactions of SB290157 with the residues involved in the hydrophobic volume and strong ionic-lock regions are shown in Fig. 7B, in which the carboxylic acid of SB290157 is stabilized by R340 (R5.42) and R161 (R4.64) and the guanine moiety of SB290157 is stabilized by D417 (D7.35). The aromatic residues F107 (F3.37), F345 (F5.47), W390 (W6.48) and Y393 (Y6.51) of human $\mathrm{C} 3 \mathrm{aR}$ stabilized the hydrophobic biaryl moiety of SB290157. Fig. 7B also shows a strong hydrogen-bond interaction between the carbonyl group of SB290157 and R340 (R5.42) of human C3aR.

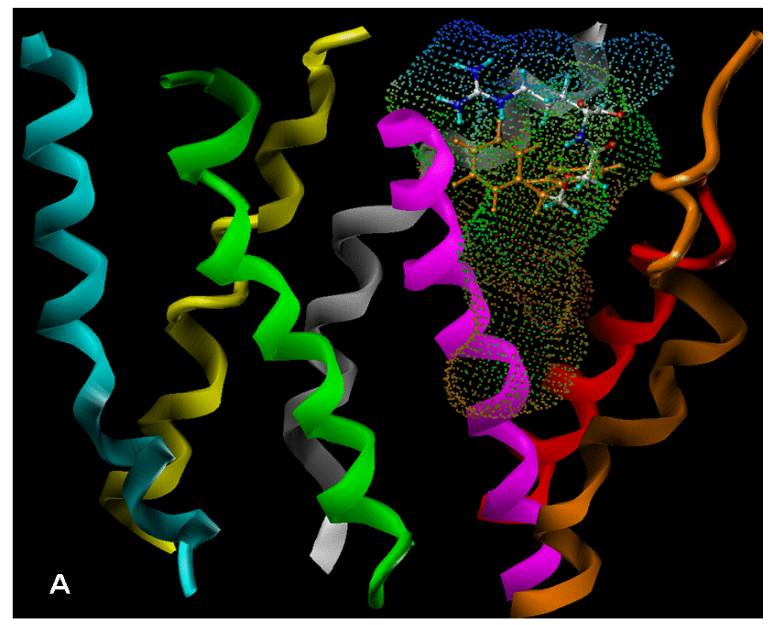

Fig. 7A- Proposed docking conformation of SB290157 (in ball and stick representation) into human $\mathrm{C} 3 \mathrm{aR}$. Lipophilic active site volume is shown in dots.

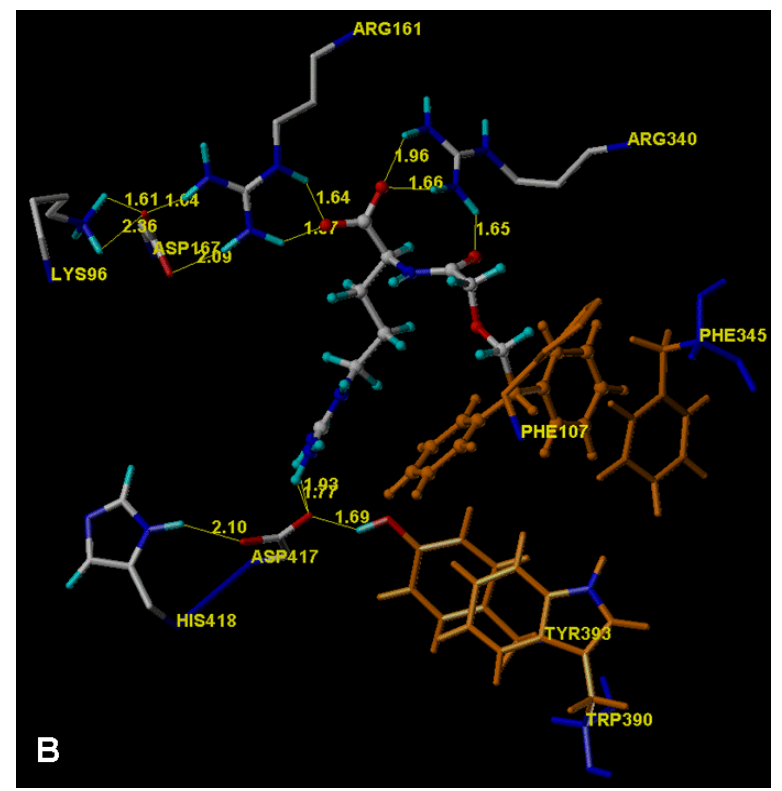

Fig. 7B- Strong ionic-lock interactions (distances in $\AA$ ) of SB290157 (ball-and-stick) with key residues (capped sticks). Hydrophobic region of the molecule is shown in brown and some hydrogens of the residues are not shown for clarity.

The stereospecificity of docking of SB280157 was explored by placing the other isomer of SB290157 (R-stereoisomer) into the $\mathrm{C} 3 \mathrm{aR}$ and undertaking similar molecular dynamics and energy minimization studies. Surprisingly, the R-isomer was found to fit more or less in the same volume and in a similar fashion as that of SB290157. The R-isomer also exhibited identical interactions with active site residues as the S-isomer (SB290157). Fig. 7C gives the inter-atomic distances not only between the residues of the active site but also the distances between these residues and the $\mathrm{R}$-isomer. An overlay of the two docked isomers with the protein 
gave an RMSD of about $1.7 \AA$, indicating that the receptor conformation does not change during the overall computational process upon docking of SB290157 into human C3aR. A closer inspection of the docked conformations of the $(R)$ and $(S)$ isomers of SB290157 indicated the presence of a major change in the 015C16-C17-018 torsion angle [158 vs. 277] as shown in Fig. 8 and a strong intramolecular hydrogen-bond between the amide $\mathrm{N}-\mathrm{H}$ and the oxygen atom in the linker of the R-isomer [rO...H = $2.3 \AA]$ and not of the S-isomer [rO...H = 3.4 $\AA$ ]. Fig. 9 illustrates the overall docking conformations of the R and S-isomers of SB290157 with the human $\mathrm{C} 3 \mathrm{aR}$. The overall binding energy difference between these two isomers was calculated to be within $5 \mathrm{Kcal} / \mathrm{mol}$ of each other indicating a nonstereospecific interaction of human C3aR with SB290157. It may be concluded, therefore, that the presence of ionic salt-bridge interactions are more important between human C3aR and SB290157 than the intramolecular hydrogen-bonding feature present within the $R$ and $S$ isomers of SB290157.

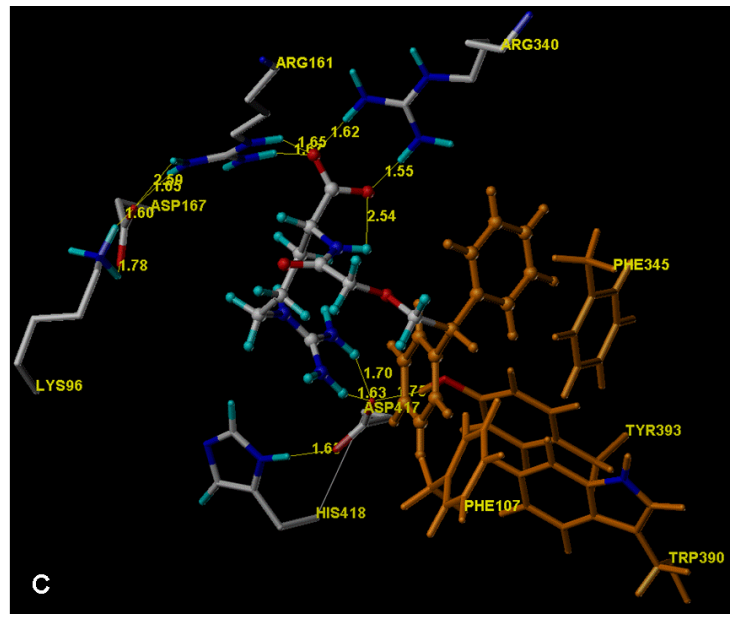

Fig. 7C- Strong ionic-lock interactions (distances in $\AA$ ) of the Risomer of SB290157 (ball-and-stick) showing key residues (capped sticks). Hydrophobic region of the molecule is shown in brown and some hydrogens of the residues are not shown for clarity.

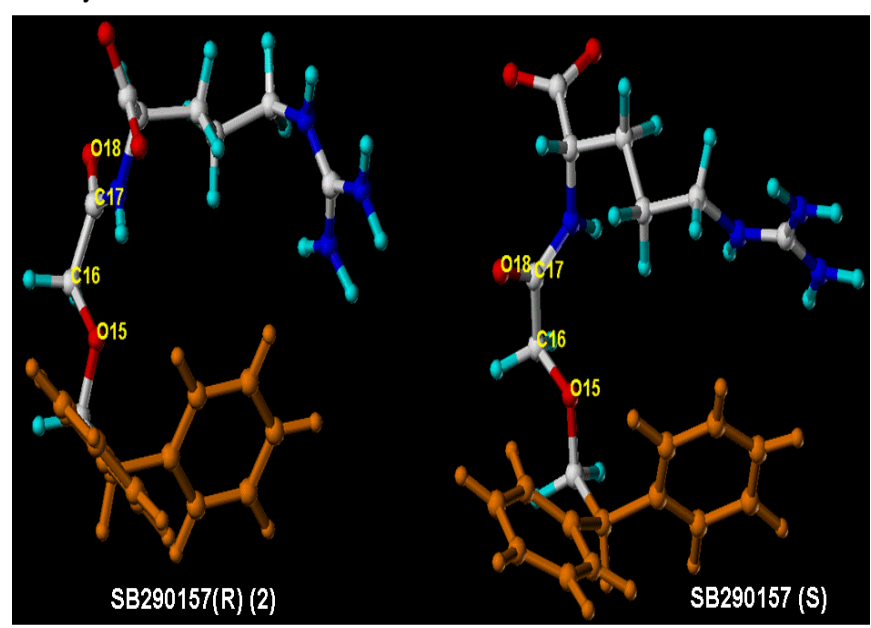

Fig. 8- Docked conformation of SB290157 and the corresponding $\mathrm{R}$-isomer in the human C3aR highlighting the key change in the dihedral angle of the labeled atoms.

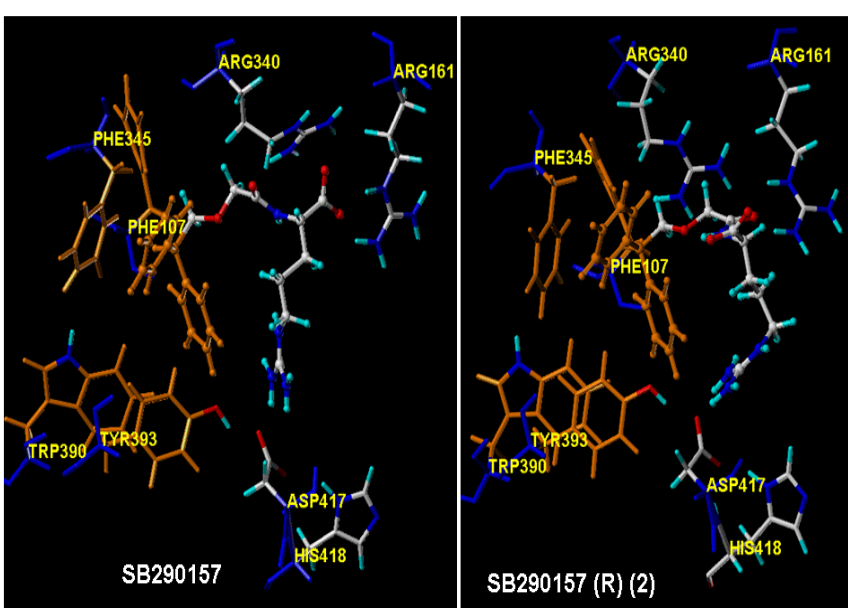

Fig. 9- Docked conformation of SB290157 and its R-isomer with human $\mathrm{C} 3 a \mathrm{R}$ highlighting key receptor residues involved in ligand binding. Ligands are displayed in ball-and-stick, while the residues are shown in capped-sticks.

\section{Conclusion}

Molecular modeling studies of a reliable homology model of C3aR have resulted in the identification of several key salt-bridge interactions at the junction of transmembrane helices with extracellular loops leading to stabilization of the receptor's structure. The active site of human C3aR was found to include ionic-lock interactions and a hydrophobic environment. The docking of SB290157 into human $\mathrm{C} 3 \mathrm{aR}$ revealed minor stereospecific ligand effects in the presence of strong ionic-lock interactions between the arginine group of the ligand and key residues of human C3aR. Our future focus will be to use the obtained results in the design of novel agonists or antagonists for C3aR as potential therapeutic agents.

\section{Acknoledgement}

The author wishes to thank lan A. Cliffe for valuable suggestions and comments.

\section{References}

[1] Porter J.E., Hwa J. and Perez D.M. (1996) J. Biol. Chem., 271 (45), 28318-28323.

[2] Porter J.E., Eldelmann S.E., Waugh D.J., Piascik M.T. and Perez D.M. (1998) Mol. Pharmacol., 53(4), 766-771.

[3] Carrieri A., Centeno N.B., Rodrigo J., Sanz F. and Carotti A. (2001) Proteins, 43(4), 382-394.

[4] Flower D.R. (1999) Biochemica et Biophysica Acta., 1422 207-234.

[5] Ta-Hsiang Chao, Ember J.A., Wang M., Bayon Y., Hugli T.E. and Ye D.R. (1999) J. Biol. Chem., 274(14), 9721-9728.

[6] Castro F.F., Schmitz-Schumann M., Rother U. and Kirschfink M. (1991) Int. Arch. Allergy Appl. Immunol., 96(4), 305-310.

[7] Kirschfink M., Castro F.F., Rother U., Nakhosteen J.A., Deppisch R. and Schmitz-Schumann M. (1993) Int. Arch. Allergy Immunol., 100(2), 151-155.

[8] Makrides S.C. (1998) Pharmacological Reviews, 50(1), 59-87.

[9] Humbles A.A., Lu B., Nilsson C.A., Lilly C., Israel E., Fujiwara Y., Gerard N.P. and Gerard C. (2000) Nature, 406, 998-1001.

[10]Krug N., Tschernig T., Erpenbeck V.J., Holfeld J.M. and Khol J. (2001) Amer. J. Respir. Crit. Care, 164, 1841-1843. 
[11]Nakano Y., Morita S., Kawamoto A., Suda T., Chida K. and Nakamura H. (2003) J. Allergy Clin. Immunol., 112(3), 525530.

[12]Ali H. and Panettieri Jr. R.A. (2005) Respiratory Research, 6 (1), 19.

[13]Thangam E.B., Venkatesha R.T., Zaidi A.K., Jordan-Sciutto K.L., Goncharov D.A., Krymskaya V.P, Amrani Y., Panettieri R.A. Jr. and Ali H. (2005) FASEB J., 19(7), 798-800.

[14]Bradding P. (2005) FASEB J., 19(12), 1585.

[15]Paques E.P., Scholze H. and Huber R. (1980) Hoppe-Seyler's Z. Physiol. Chem., 361(6), 977-979.

[16]Huber R., Scholze H., Paques E.P. and Deisenhofer J. (1980) Hoppe-Seyler's Z. Physiol. Chem., 361(9), 1388-1389.

[17]Unson C.G., Erickson B.W. and Hugli T.E. (1984) Biochemistry, 23(4), 585-589.

[18]Bokisch V.A. and Muller-Eberhard H.J. (1970) J. Clin. Invest., 49(12), 2427-2436.

[19]Chazin W.J., Hugli T.E. and Wright P.E. (1988) Biochemistry, 27(26), 9139-9148.

[20]Sun J., Ember J.A., Chao TA-Hsiang, Fukuoka Y., Ye R.D., Hugli T.E. (1999) Protein Science, 8, 2304-2311.

[21]Ames R.S., Lee D., Foley J.J., Jurewicz A.J., Tornetta M.A., Bautsch W., Settmacher B., Klos A., Erhard K.F, Cousins R.D., Sulpizio A.C., Hieble J.P., McCafferty G., Ward K.W., Adams J.L., Bondinell W.E., Underwood D.C., Osborn R.R., Badger A.B. and Sarau H.M. (2001) J. Immunol. 166(10), 6341-6348.

[22]Mathieu M.C., Sawyer N., Greig G.M., Hamel M., Kargman S., Ducharme Y., Lau C.K., Friesen R.W., O'Neill G.P., Gervais F.G. and Therien A.G. (2005) Immunol. Lett., 100(2), 139-145.

[23]Palczewski K., Kumasaka T., Hori T., Behnke C.A., Motoshima H., Fox B.A., Le Trong I., Teller D.C., Okada T., Stenkamp R.E., Yamamoto M. and Miyano M. (2000) Science 289, 739745.

[24]Ballesteros J.A. and Weinstein H. (1995) Methods Neurosci., 25, 366-428.

[25]Visiers I., Ballesteros J.A. and Weinstein H. (2002) Meth. Enzymol., 343, 329-371.

[26]SYBYL7.0; Tripos Inc., 1699 South Hanley Rd, St. Louis, Missouri, 63144.

[27]Spalsing T.A., Burstein E.S., Henderson S.C., Ducote K.R. and Brann M.R. (1998) J. Biol. Chem. 273(34), 21563-21568.

[28]Visiers I., Ebersole B.J., Dracheva S., Ballesteros J., Sealfon S.C. and Weinstein H. (2002) Int. J. Quant. Chem., 88 (1), 6575. 